Fakultät III

Wirtschaftswissenschaften, Wirtschaftsinformatik und Wirtschaftsrecht

Volkswirtschaftliche Diskussionsbeiträge

Discussion Papers in Economics

No. $151-11$

November 2011

Thomas Eichner · Rüdiger Pethig

Flattening the carbon extraction path in unilateral costeffective action 
Universität Siegen

Fakultät III

Wirtschaftswissenschaften, Wirtschaftsinformatik und Wirtschaftsrecht

Fachgebiet Volkswirtschaftslehre

Hölderlinstraße 3

D-57068 Siegen

Germany

http://www.uni-siegen.de/fb5/vwl/

ISSN 1869-0211

Available for free from the University of Siegen website at http://www.uni-siegen.de/fb5/vwl/research/diskussionsbeitraege/

Discussion Papers in Economics of the University of Siegen are indexed in RePEc and can be downloaded free of charge from the following website:

http://ideas.repec.org/s/sie/siegen.html 


\title{
Flattening the carbon extraction path in unilateral cost-effective action*
}

\author{
Thomas Eichner \\ Department of Economics, University of Hagen \\ Rüdiger Pethig \\ Department of Economics, University of Siegen
}

\begin{abstract}
Internalizing the global negative externality of carbon emissions requires flattening the extraction path of world fossil energy resources (= world carbon emissions). We consider governments having sign-unconstrained emission taxes at their disposal and seeking to prevent world emissions from exceeding some binding aggregate emission ceiling in the medium term. Such a ceiling policy can be carried out either in full cooperation of all (major) carbon emitting countries or by a sub-global climate coalition. Unilateral action has to cope with carbon leakage and high costs which makes a strong case for choosing a policy that implements the ceiling in a cost-effective way. In a two-country two-period general equilibrium model with a non-renewable fossilenergy resource we characterize the unilateral cost-effective ceiling policy and compare it with its fully cooperative counterpart. We show that with full cooperation there exists a cost-effective ceiling policy in which only first-period emissions are taxed at a rate that is uniform across countries. In contrast, the cost-effective ceiling policy of a sub-global climate coalition is characterized by emission regulation in both periods. That policy may consist either of positive tax rates in both periods or of negative tax rates $(=$ subsidies) in both periods or of a positive rate in the first and a negative rate in the second period. The share of the total stock of energy resources owned by the sub-global climate coalition turns out to be a decisive determinant of the sign and magnitude of unilateral cost-effective taxes.
\end{abstract}

JEL-classification: H22, Q32, Q54

Keywords: $\quad$ unilateral climate policy, intertemporal climate policy non-renewable energy resources, emission taxes

*Eichner: Department of Economics, University of Hagen, Universitätsstr. 41, 58097 Hagen, Germany, email: thomas.eichner@fernuni-hagen.de; Pethig: Department of Economics (Fakultät III), University of Siegen, Hölderlinstr. 3, 57068 Siegen, Germany, email: pethig@vwl.wiwi.uni-siegen.de 


\section{The problem}

Scientific evidence strongly suggests that global warming is caused by anthropogenic greenhouse gas emissions, notably by emissions of carbon dioxide from burning fossil fuels. ${ }^{1}$ Carbon emissions create allocative distortions and global welfare losses in the absence of climate policy, because emissions generate external costs rendering sub-optimal the laissezfaire time path of emissions. The shape of welfare enhancing emission pathways depends on various factors such as stock-dependent extraction costs, low-carbon resource substitutes, the severity of climate damage, and the size of discount rates. The important question whether reductions in the present value of welfare losses suffered in laissez faire are or are not feasible without reducing cumulative emissions cannot be easily answered on empirical grounds. In the model to be analyzed here the world stock of fossil energy is given and will be fully depleted in the laissez-faire world economy. Moreover, we share the view that "... public policies that would limit the overall extraction and exempt part of the stock in situ permanently from extraction ... seem hardly defendable" (Sinn 2008, p. 376)2 because renewables are imperfect substitutes for fossil fuels and because a demand for the latter can always be expected even if extraction costs are increasing in cumulative extraction (and a fortiori under the simplifying assumption of zero extraction costs). ${ }^{3}$

There is scope for welfare-enhancing climate policies that do not limit overall extraction for the following reason. Recent climate research (Allen et al. 2009, Meinshausen et al. 2009) suggests (i) that there is a strong correlation between the maximum level of warming and cumulative emissions and (ii) that peak warming does not depend on the shape of the emission pathway. These findings do not imply, however, that the time path of warming up to the peak and beyond is independent of the emission pathway. Clearly, the flatter is the time path of emissions - keeping cumulative emissions fixed - the later the peak of warming will be attained. As damage from global warming is increasing in the mean temperature, standard welfare economics suggests that in the laissez-faire scenario too much carbon is emitted too early. Hence flattening the emission pathway is welfare enhancing for the entire world, and some (presumably more moderate) flattening is then also welfare enhancing for a sub-global climate coalition when the rest of the world abstains from climate policy.

\footnotetext{
${ }^{1}$ In the present paper we disregard greenhouse gases other than carbon dioxide.

${ }^{2}$ Hoel (2010) and Michielsen (2011) also assume that an exogenously given stock of fossil fuel is fully exhausted.

${ }^{3}$ There is another strand of literature which focuses on climate policy instruments capable to impact on the level of cumulative extraction of fossil fuel resources. See e.g. Van der Ploeg and Withagen (2010), Grafton et al. (2010), Kalkuhl and Edenhofer (2010) and Gerlagh (2011).
} 
In our subsequent analysis we do not explicitly model these welfare-economic considerations although the motivation for the study of those global or sub-global climate policies which flatten the laissez-faire extraction/emission path is based on the arguments outlined above. To further simplify we envisage a global or sub-global climate coalition that seeks to prevent cumulative world emissions from exceeding some ceiling in the medium term, say 2050, which is tighter than the global cumulative emissions until 2050 in the absence of climate policy. Its implementation therefore requires a proactive policy approach which we denote as ceiling policy, for short.

We study ceiling policies of an international sub-global climate coalition in an effort to account in a very stylized way, though, for the state and perspectives of the ongoing international climate negotiations. The political goal of keeping the world mean temperature from rising in the medium term by $2^{\circ}$ Celsius or more above preindustrial levels has been endorsed by numerous governments and most recently also by the UN Conference of the Parties in Cancun (UNFCCC 2010). We interpret that as the acknowledgement of a target to flatten the world carbon extraction path. If an international agreement will be reached at all in the future, it will likely be on a medium term emission ceiling. Yet in view of the poor progress made in international climate negotiations over the last decades, an agreement encompassing all (major) countries can hardly be expected. That is why our emphasis is on ceiling policies of a sub-global climate coalition (which needs to be larger than the European Union) while the grand coalition serves as a benchmark only. The countries in such a sub-global coalition are supposed to follow a cooperative approach and implement a medium term ceiling they have agreed upon. That is far more than what we observe at present, where various individual countries carry out - or have announced to carry out - some carbon mitigation policies. The pledges have been made without international coordination and without binding commitments which is why the net effect of all these non-cooperative national actions on world emissions is unclear.

In the present paper we envisage a sub-global climate coalition and presuppose that there exist feasible ceiling policies for that coalition (which can safely be assumed, if the coalition is not too small and the ceiling is not too tight). The focus is then on characterizing the set of feasible ceiling policies and on investigating properties of that particular feasible ceiling policy which achieves the predetermined ceiling at minimum cost for the coalition. For reference purposes we will also compare the cost-effective ceiling policy of the sub-global coalition to the fully cooperative cost-effective ceiling policy.

This paper is related to the literature on carbon leakage which arises when one country's unilateral emission reduction policy increases the emissions in other countries. The 
so-called green paradox (Sinn 2008, Eichner and Pethig 2011) is said to occur in the extreme case in which unilateral emission reductions increase rather than reduce aggregate world emissions, as compared to their level in the absence of that unilateral policy. ${ }^{4}$ Hoel (1991), Bohm (1993), Golombek and Hoel (2004), Copeland and Taylor (2005), Di Maria and van der Werf (2005), Ishikawa and Kiyono (2006), van der Ploeg and Withagen (2009), Eichner and Pethig (2011) have analytically explored various channels and determinants of carbon leakage and/or the green paradox. The green paradox is not an issue in the present paper, because by presupposition, the unilaterally acting sub-global climate coalition avoids excessive leakage by choosing appropriate tax rates in all periods. In that respect our approach is closer to Chakravorty et al. (2006) and Kalkuhl and Edenhofer (2010) who employ the ceiling or carbon budget approach and characterize the cost-effective (cooperative) carbon budget policy. However, these studies employ one-country growth models. To our knowledge the extant literature does not consider sub-global climate coalitions pursuing a policy of limiting cumulative medium-term emissions.

We will carry out the analysis in a stylized two-country two-period model similar to that in Eichner and Pethig (2011). Each country owns a share of the finite world stock of fossil-energy resource. Governments are assumed to have at their disposal emission taxes in both periods. The carbon ceiling limits both countries' overall first-period emissions and is binding, i.e. is fixed below the countries' overall first-period emissions in the laissezfaire economy. First we characterize the fully cooperative cost-effective ceiling policy as a benchmark. It turns out that in this case cost effectiveness can be achieved through a ceiling policy in which only first-period emissions are taxed at a rate that is uniform across countries. That policy is in the spirit of results from dynamic one-country models (e.g. Sinclair 1992, 1994, Sinn 2008) in which flattening the fossil-fuel extraction path requires high emission taxes early on and low or no taxes later.

Next we investigate the case of unilateral ceiling policies where the sub-global climate coalition is represented by one of the countries in our two-country model. In its effort to meet the ceiling in unilateral action that country's challenge is to restrict total firstperiod emissions via its domestic emission taxes which have an impact only on domestic emissions in both periods and thus fail to have full control over the ceiling. Knowing that the government of the other country abstains from climate policy, the active country carries out its ceiling policy strategically in the sense that it takes into account the responses to its tax policy of all domestic and foreign consumers and firms. We show that there is a large

\footnotetext{
${ }^{4}$ There are various related concepts of green paradox, e.g. "... that anticipation of future reductions in demand for oil and other fossil fuels will drive the resource owners to bring forward their supply." (Gerlagh 2011).
} 
set of feasible ceiling policies for one and the same predetermined ceiling (under the implicit assumption that the ceiling is not too tight) and we classify these policies with respect to the sign and magnitude of tax rates and with respect to the prices of fossil fuel and the consumption good corresponding to each policy. There are feasible policies with positive tax rates (emission taxes proper) in both periods, with negative tax rates (subsidies) in both periods, and there are feasible policies with a positive tax rate in the first and a negative rate in the second period. All these policies differ, of course, with respect to the cost $(=$ welfare loss) accruing to the country that undertakes the unilateral action. It is therefore of great interest to identify the least-cost ceiling policy among the feasible policies. We find that the sign and magnitude of tax rates constituting the cost-effective unilateral ceiling policy depend on the distribution of ownership of the stock of fossil energy. The larger the resource stock of the country is that implements the ceiling unilaterally, the larger is the shift of first and second-period tax rates from positive to negative rates.

The paper is organized as follows. Section 2 outlines the model. Section 3 briefly investigates the properties of the cooperative cost-effective ceiling policy and characterizes unilateral ceiling policies. We focus on feasibility of unilateral ceiling policies in the first part and on cost effectiveness of unilateral ceiling policies in the second part of Section 3. Section 4 concludes.

\section{The competitive two-country economy with carbon ceiling regulation}

The structure of the model. In period $t=1,2$ country $i=A, B$ produces the amount $x_{i t}^{s}$ of the consumption good $X$, using fossil fuel $e_{i t}$ as an input, according to

$$
x_{i t}^{s}=X^{i}\left(e_{i t}\right)
$$

The representative consumer in country $i$ derives utility,

$$
u_{i}=U^{i}\left(x_{i 1}, x_{i 2}\right),
$$

from consuming the amount $x_{i t}$ of good $X$ in period $t$. Fossil fuel is a non-renewable resource. Its total stock is $\bar{e}$ and country $i$ owns the share $\alpha_{i}(i=A, B)$ of that stock, where $\alpha_{A}=\left(1-\alpha_{B}\right) \in[0,1]$.

Carbon emissions are generated in strict proportion to the amount of fossil fuel consumed. Hence with suitable definitions of units, $e_{i t}$ denotes fuel consumption as well as 
carbon emissions. The supply constraints for fossil fuel and for the consumption good $X$,

$$
\begin{aligned}
\bar{e} & =e_{A 1}+e_{B 1}+e_{A 2}+e_{B 2}, \\
x_{A t}^{s}+x_{B t}^{s} & =x_{A t}+x_{B t} \quad t=1,2
\end{aligned}
$$

are obvious feasibility requirements. They turn into world market equilibrium conditions in the competitive economy studied below.

Regulation, competitive markets, and the agents' optimization problems. The principal target of regulation is to keep total first-period emissions from exceeding an upper bound $\bar{e}_{1}>0$, which translates into the constraint

$$
\bar{e}_{1}=e_{A 1}+e_{B 1}
$$

We refer to $\bar{e}_{1}$ as (carbon) ceiling, for short. By ruling out the greater sign in the constraint (5) we restrict attention to ceilings $\bar{e}_{1}$ that are smaller than total first-period emissions in the absence of regulation. ${ }^{5}$ Consequently, some fossil-fuel consumption needs to be shifted from the first to the second period as compared with the laissez-faire scenario. That is exactly what the ceiling policy is about.

To meet the ceiling, the governments of both countries have the option of regulating their domestic carbon emissions in either period. They can do so in two conceptually equivalent ways. Either they introduce national cap-and-trade schemes in one or both periods, where the emission cap of country $i$ in period $t$ is the politically chosen level of $e_{i t}$ and where $\pi_{i t}$ is the corresponding permit price. In that case, the independent policy variable is the cap $e_{i t}$ and the permit price adjusts as to equilibrate the permit market. Alternatively, we interpret $\pi_{i t}$ as the rate of an emission tax country $i$ levies in period $t$. In that case, the tax is the independent policy variable the regulator chooses such that the resultant endogenous emissions $e_{A 1}$ and $e_{B 1}$ meet the ceiling $\bar{e}_{1}$. However, in the present context these alternative interpretations are not equivalent because scenarios with 'negative emission prices' $\pi_{i t}$ for some $i$ and $t$ will turn out to be relevant. As negative prices cannot emerge as permit market equilibrium prices we stick to the tax policy interpretation in the sequel with the understanding that a negative tax is a subsidy. ${ }^{6}$

We will focus on two policy scenarios. In the first benchmark case both countries cooperate and coordinate their tax policies to implement the ceiling. In the second scenario

\footnotetext{
${ }^{5}$ The limiting case of the ceiling being equal to laissez-faire emissions will turn out to be of some interest in Section 4.

${ }^{6}$ To avoid complicated wording we refer to $\pi_{i t}$ as a tax rate except in specific results where we have explicitly established that $\pi_{i t}<0$.
} 
country $B$ refrains from taxing emissions altogether $\left(\pi_{B 1}=\pi_{B 2} \equiv 0\right)$ while country $A$ proceeds to meet the ceiling in unilateral action.

The ceiling policy is embedded in a perfectly competitive two-country economy. In each period $t=1,2$ there exists a world market for the consumption good $X$ with price $p_{x t}$ and a world market for fossil fuel with price $p_{e t}$. We take the consumption good $X$ in period 1 as numéraire, $p_{x 1}=1$, and write $p_{x 2}=p_{x}$ for convenience of notation.

Although productive capital is not modeled, there is a financial market for consumer savings and loans. As shown in the Appendix A, that credit market is cleared at any fixed interest rate, $r$, if the second-period markets for energy and the consumption good are in equilibrium. For analytical convenience we normalize the interest rate to be zero $(r \equiv 0){ }^{7}$ In each country $i$ a resource firm extracts the domestic stock of fossil fuel, $\bar{e}_{i}=\alpha_{i} \bar{e}$, over both periods. With zero extraction costs assumed we simplify the exposition by considering one 'aggregate' resource firm (rater than two separate firms) the share $\alpha_{i}$ of which is owned by country $i$. That firm extracts the entire stock of fossil fuel, $\bar{e}$, and its present value profits are $p_{e 1} e_{1}+\frac{p_{e 2} e_{2}}{1+r}=\sum_{t} p_{e t} e_{t}$ (where the last term follows from $r \equiv 0$ ). The resource firm maximizes its present value profits subject to $e_{1}+e_{2}=\bar{e}$ which yields the simple Hotelling rule $p_{e 1}=p_{e 2}=: p_{e}$. As country $i$ owns the share $\alpha_{i}$ of the resource stock, it claims the share $\alpha_{i}$ of the firm's profits.

The representative consumer of country $i$ maximizes utility $U^{i}\left(x_{i 1}, x_{i 2}\right)$ subject to the consolidated budget constraint ${ }^{8} x_{i 1}+\frac{p_{x} x_{i 2}}{1+r}=x_{i 1}+p_{x} x_{i 2}=: y_{i}$, where country $i$ 's present value of total income is

$$
\begin{aligned}
y_{i} & =\left[x_{i 1}^{s}-\left(p_{e}+\pi_{i 1} e_{i 1}\right)\right]+\frac{p_{x} x_{i 2}^{s}-\left(p_{e}+\pi_{i 2}\right) e_{i 2}}{1+r}+\left[\pi_{i 1} e_{i 1}+\frac{\pi_{i 2} e_{i 2}}{1+r}\right] \\
& =x_{i 1}^{s}+p_{x} x_{i 2}^{s}+p_{e} \Delta e_{i} \quad \text { with } \Delta e_{i}:=\alpha_{i} \bar{e}-e_{i 1}-e_{i 2} \quad i=A, B .
\end{aligned}
$$

Utility maximization yields

$$
\frac{U_{x_{i 2}}^{i}}{U_{x_{i 1}}^{i}}=p_{x} \quad i=A, B .
$$

In each country $i$ an aggregate price-taking firm produces the consumption good $X$. Maximizing profits $\sum_{t}\left[p_{x t} X\left(e_{i t}\right)-\left(p_{e t}+\pi_{i t}\right) e_{i t}\right]$ for $i=A, B$ gives us the first-order

\footnotetext{
${ }^{7}$ In many growth models an alternative but equivalent procedure is applied: The prices of the consumption good are set equal to one in all periods (here $p_{x 1}=p_{x 2}=1$ ) while the interest rate $r$ is determined endogenously.

${ }^{8}$ The last bracketed term $\left[\pi_{i 1} e_{i 1}+\pi_{i 2} e_{i 2} /(1+r)\right]$ is the (positive or non-positive) tax payments of the producer of the consumption good in country $i$ (see below) which is recycled to the consumer.
} 
conditions

$$
\begin{array}{rlrl}
X_{e_{i 1}}^{i} & =p_{e}+\pi_{i 1} & i=A, B, \\
p_{x} X_{e_{i 2}}^{i} & =p_{e}+\pi_{i 2} & i=A, B .
\end{array}
$$

\section{Unilateral carbon ceiling regulation}

In the present section we assume that country $A$ seeks to implement the ceiling $\bar{e}_{1}$ unilaterally knowing that country $B$ is non-cooperative and refrains from climate policy altogether. In general, lack of cooperation is not tantamount to climate policy inactivity (unless country $B$ denies the impact of anthropogenic carbon emissions on the climate) because in the absence of cooperation Nash behavior (best reply) would be in the interest of country $B$ 's government. However, assuming Nash behavior for country $B$ 's government would require introducing climate damage into the formal model which in turn would impede analytical tractability and informative results. Apart from that 'technical' argument we do not see compelling reasons why the results to be derived below under the assumption of country $B$ 's inactivity should differ qualitatively from those of an extended (numerical) model in which the government of country $B$ plays Nash.

Before we study country $A$ 's unilateral ceiling policy it is useful to briefly investigate the fully cooperative cost-effective ceiling policy for the purpose of later comparison. Suppose both countries join forces and aim at implementing the ceiling $\bar{e}_{1}$ at minimum total welfare cost $w_{0}-w_{1}$, where $w=\omega_{A} u_{A}+\omega_{B} u_{B}$ is world welfare with agreed-upon positive welfare weights ${ }^{9} \omega_{A}$ and $\omega_{B}$ and where $w_{0}$ and $w_{1}$, respectively, is world welfare ${ }^{10}$ before and after the cooperative ceiling policy. In order to characterize analytically the cooperative cost-effective ceiling policy, consider a social planner who maximizes world welfare $\sum_{i=A, B} \omega_{i} u_{i}$ subject to (1) - (5). The first-order conditions of the corresponding Lagrangean determine the efficient allocation and the standard procedure of decentralization by prices (and taxes), as outlined in the Appendix B, results in

\section{Proposition 1.}

(i) The cooperative tax policy $\left(\pi_{A 1}, \pi_{A 2}, \pi_{B 1}, \pi_{B 2}\right)$ implements the ceiling $\bar{e}_{1}$ cost-effectively, if $\pi_{A 2}=\pi_{B 2}=0$ and $\pi_{A 1}=\pi_{B 1}=\bar{\mu}$, where $\bar{\mu}$ is the shadow price of the ceiling constraint $\bar{e}_{1}=e_{A 1}+e_{B 1}$.

\footnotetext{
${ }^{9}$ The welfare weights can be interpreted as being fixed in a cost-sharing agreement which is taken as given.

${ }^{10}$ World welfare is calculated here before environmental damage has been subtracted.
} 
(ii) The implementation of the ceiling distorts the allocation (compared to the no-policy equilibrium) by driving a wedge between the marginal rates of intertemporal substitution in production and consumption:

$$
\frac{U_{x_{i 2}}^{i}}{U_{x_{i 1}}^{i}}-\frac{X_{e_{i 1}}^{i}}{X_{e_{i 2}}^{i}}=\frac{\bar{\mu} p_{x}}{p_{e}} \quad \text { for } \quad i=A, B
$$

According to Proposition 1(i) the cost-effective implementation of the ceiling is achieved by a policy that leaves second-period emissions unregulated and levies a tax on first-period emissions that is uniform across countries ${ }^{11}$ and reflects the stringency of the ceiling. The wedge (10) is also uniform across countries ${ }^{12}$ and is the only distortion caused by the cooperative ceiling policy. The Appendix B shows that the cooperative ceiling policy satisfies consumption efficiency, (inter- and intra-period) production efficiency and in view of (10) the ceiling drives a wedge between the marginal rate of intertemporal substitution and the ratio of inter-period marginal productivities of good $X$.

For the sake of completeness we note that the policy identified in Proposition 1(i) is not the only cost-effective ceiling policy. As shown in the Appendix B there is a large set of policies with non-zero (and even negative) tax rates that all support the unique efficient allocation. Since the policy of Proposition 1(i) is the simplest of all these cost-effective policies we will disregard the others.

Having characterized as a benchmark the cooperative cost-effective ceiling policy we now turn to country $A$ 's unilateral ceiling policy. To that end we need to presuppose the existence of feasible strategies for country $A$ to implement $\bar{e}_{1}$ unilaterally. ${ }^{13}$ In its effort to meet the ceiling $\bar{e}_{1}$ in unilateral action country $A$ 's challenge is to restrict total first-period emissions to $\bar{e}_{1}$ via its tax rates $\left(\pi_{A 1}, \pi_{A 2}\right)$ which have an impact on the national emissions $e_{A 1}$ and $e_{A 2}$ but do not determine the ceiling directly. Knowing that the government of country $B$ is inactive, country $A$ will be assumed to account for the responses to its tax policy of the consumers and firms in both countries.

Unfortunately, informative results cannot be derived in the model used so far with general production functions $X^{i}$ and utility functions $U^{i}$. To make progress we will reduce complexity in the remainder of the paper by assuming that the functions $X^{i}$ and $U^{i}$ are

\footnotetext{
${ }^{11}$ Rather than levying uniform national taxes in the first period, one could also introduce a uniform world-wide tax in each period and use the proceeds for meeting burden-sharing requirements.

${ }^{12}$ The feature that the wedge is the same across countries keeps the distortion small and will not carry over to the case of unilateral ceiling policy to be studied later.

${ }^{13}$ For more details see Section 3.1 below.
} 
the same for both countries and that they take on the parametric forms

$$
\begin{array}{ll}
x_{i t}^{s}=X^{i}\left(e_{i t}\right)=a e_{i t}-\frac{b}{2} e_{i t}^{2}, & a, b>0, i=A, B, t=1,2, \\
u_{i}=U^{i}\left(x_{i 1}, x_{i 2}\right)=x_{i 1}^{\gamma} x_{i 2}^{1-\gamma} & \gamma \in] 0,1[, i=A, B .
\end{array}
$$

As a consequence, (3), (5), (11) and the equivalent of (8) and (9) yield the fuel demand functions

$$
\begin{aligned}
& e_{A 1}=\frac{a}{b}-\frac{p_{e}}{b}-\pi_{1}, \quad e_{A 2}=\frac{a}{b}-\frac{p_{e}}{b p_{x}}-\pi_{2}, \quad \text { with } \quad \pi_{1}:=\frac{\pi_{A 1}}{b} \quad \text { and } \pi_{2}:=\frac{\pi_{A 2}}{b p_{x}}, \\
& e_{B 1}=\bar{e}_{1}-e_{A 1}=\frac{a}{b}-\frac{p_{e}}{b}, \quad e_{B 2}=\bar{e}_{2}-e_{A 2}=\frac{a}{b}-\frac{p_{e}}{b p_{x}} .
\end{aligned}
$$

It is convenient to replace the 'original' emission tax rates $\pi_{A 1}$ and $\pi_{A 2}$ by the modified $\operatorname{tax}$ rates $\pi_{1}$ and $\pi_{2}$ throughout the rest of the paper. The appealing implication of this substitution is the observation that $\pi_{t}=e_{B t}-e_{A t}$, i.e. that $\pi_{t}$ is a direct measure of the (intra-period) production distortion in period $t$.

The commodity demand functions

$$
x_{i 1}=\gamma y_{i} \quad \text { and } \quad x_{i 2}=\frac{(1-\gamma) y_{i}}{p_{x}} \text { for } i=A, B
$$

follow from (6), (7) and (12), after some rearrangement of terms. We conclude that under the functional forms (11) and (12) the competitive equilibria with unilateral ceiling policy are fully characterized by the 12 equations (4), (6), (13), (14) and (15) which contain the 12 variables $^{14} e_{A 1}, e_{A 2}, p_{e}, p_{x}, x_{A 1}, x_{A 2}, x_{B 1}, x_{B 2}, y_{A}, y_{B}, \pi_{1}$ and $\pi_{2}$. According to Walras Law, one of the market clearing conditions in (4) is already implied by all other equations. Thus we are left with 11 equations for 12 variables. As an implication, country $A$ can choose among a variety of ceiling policies for any predetermined (not too stringent) ceiling. The existence of multiple ceiling policies is, of course, a precondition for both the opportunity and the need to select a cost-effective policy.

\subsection{Characterization of feasible unilateral ceiling policies}

To prepare for the analysis of cost-effective unilateral ceiling policies, it is useful to first explore the properties of unilateral feasible ceiling policies. As shown in the preceding section we have a degree of freedom in specifying unilateral ceiling policies and we will exploit that discretion by investigating the properties of the set of feasible ceiling policies generated by alternative 'fixed' levels of $e_{A 1} \cdot{ }^{15}$ Consider a strictly binding ceiling $\bar{e}_{1}$ and

\footnotetext{
${ }^{14}$ The outputs $x_{i t}^{s}$ are already eliminated via (11) and the inputs $e_{B 1}$ and $e_{B 2}$ via (3) and (5).

${ }^{15}$ Technically speaking, one could have taken as exogenous any other variable.
} 
denote by $E$ the set of all $e_{A 1}$ for which a ceiling policy exists, i.e. for which the equations (4), (6), (13), (14) and (15) have a solution. Observe that $e_{A 1}=0$ is a potential candidate ('at the limit') for supporting a ceiling policy because $p_{e}$ can take on the (low) level $\breve{p}_{e}:=a-b \bar{e}_{1}$ at which $e_{B 1}=\bar{e}_{1}$ and $\pi_{1}$ can be set high enough to push $e_{A 1}$ to zero. ( $p_{e}<\breve{p}_{e}$ is incompatible with ceiling policies because $e_{B 1}$ would then exceed $\bar{e}_{1}$.) $e_{A 1}>\bar{e}_{1}$ is no feasible choice because the extreme case $e_{A 1}=\bar{e}_{1}$ requires a level of $p_{e}$ greater than, or equal to, the choke price $p_{e}=a$. As a consequence, $E \subset\left[0, \bar{e}_{1}\right]$. It is clear that in unilateral action country $A$ can implement those ceilings that require only small reductions of the first-period emissions prevailing in the laissez-faire economy. The more stringent the ceiling is and the 'smaller' country $A$ is relative to country $B$, the smaller will be the set of feasible unilateral ceiling policies.

It is convenient to introduce the following additional notation. Define as $e_{A 1}\left(\pi_{t}=0\right)$ the value of $e_{A 1}$ that supports that particular ceiling policy which exhibits $\pi_{t}=0$ for $t=1,2$. Since the production functions are the same across countries, the ceiling policy equilibrium for $e_{A 1}\left(\pi_{t}=0\right)$ is also characterized by $e_{A t}=\bar{e}_{t} / 2$. In addition, we combine the emissions $e_{A 1}\left(\pi_{t}=0\right) \in E$ for $t=1,2$ with the interval $\left[0, \bar{e}_{1}\right]$ via the definition of the following sets

$$
\begin{aligned}
& \left.E_{\ell}:=\left\{e_{A 1} \mid 0 \leq e_{A 1}<e_{A 1}\left(\pi_{2}=0\right)\right\}, \quad E_{m}:=\right] e_{A 1}\left(\pi_{2}=0\right), \bar{e}_{1} / 2[\text { and } \\
& E_{h}:=\left\{e_{A 1} \mid \bar{e}_{1} / 2<e_{A 1} \leq \bar{e}_{1}\right\} .
\end{aligned}
$$

Note that the sets $E_{\ell},\left\{e_{A 1}\left(\pi_{2}=0\right)\right\}, E_{m},\left\{\bar{e}_{1} / 2\right\}$ and $E_{h}$ form a partition of $\left[0, \bar{e}_{1}\right]$, if and only if $e_{A 1}\left(\pi_{2}=0\right) \leq \bar{e}_{1} / 2$. We will show below that this condition is satisfied, indeed, and that the partition is useful for characterizing the set of feasible ceiling policies, $E$. With this notation we prove in the Appendix $\mathrm{C}$ the analytical properties of feasible ceiling policies and summarize the results in

Proposition 2. Suppose the ceiling satisfies $\bar{e}_{1} \leq e_{1}^{0}$, where $e_{1}^{0}$ are total first-period emissions in the laissez-faire economy.

(i) $E_{m}\left\{\begin{array}{l}\neq \\ =\end{array}\right\} \emptyset \quad \Longleftrightarrow \quad e_{A 1}\left(\pi_{2}=0\right)\left\{\begin{array}{l}< \\ =\end{array}\right\} e_{A 1}\left(\pi_{1}=0\right)=\frac{\bar{e}_{1}}{2} \quad \Longleftrightarrow \quad \bar{e}_{1}\left\{\begin{array}{l}< \\ =\end{array}\right\} e_{1}^{0}$. Moreover, $\bar{e}_{1}<e_{1}^{0}$ implies $E_{m} \subset E$.

(ii) Over the entire domain $E \subset\left[0, \bar{e}_{1}\right]$ of feasible policies, $\pi_{1}$ and $\pi_{2}$ are strictly decreasing 
in $e_{A 1}$, and the ceiling policy $\left(\pi_{1}, \pi_{2}\right)$ is characterized by
(a) $\pi_{1}>0, \pi_{2}>0$
if $e_{A 1} \in E_{\ell}$,
(b) $\pi_{1}>0, \pi_{2}=0$
if $e_{A 1}=e_{A 1}\left(\pi_{2}=0\right)$,
(c) $\pi_{1}>0>\pi_{2}$
if $e_{A 1} \in E_{m}$,
(d) $\pi_{1}=0, \pi_{2}<0$
if $e_{A 1}=e_{A 1}\left(\pi_{1}=0\right)=\bar{e}_{1} / 2$,
(e) $\pi_{1}<0, \pi_{2}<0$
if $e_{A 1} \in E_{h}$.

(iii) Over the entire domain $E \subset\left[0, \bar{e}_{1}\right]$ of feasible policies, $e_{A 2}$ and $p_{e}$ are strictly increasing in $e_{A 1}$. Over the subdomain $\left[e_{A 1}\left(\pi_{2}=0\right), \bar{e}_{1} / 2\right]$ of $E, p_{x}$ is strictly increasing in $e_{A 1}$.

(iv) Over the subdomain $\left[0, e_{A 1}\left(\pi_{2}=0\right)\right] \cap E$ of $E$, the prices $p_{e}$ and $p_{x}$ are lower than their counterparts $p_{e}^{0}$ and $p_{x}^{0}$ in the laissez-faire economy, if $\bar{e}_{1}<e_{1}^{0}$.

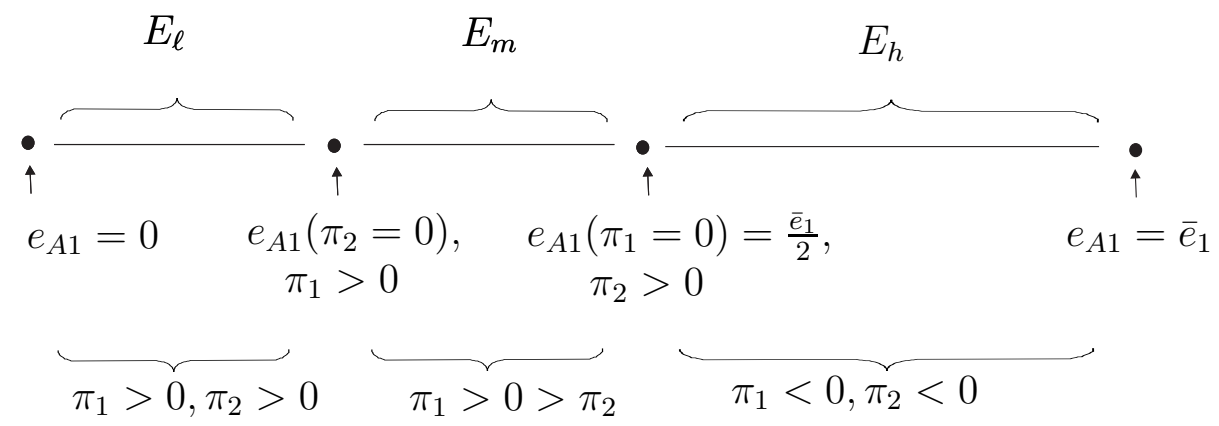

Figure 1: Unilateral ceiling policies and the domain $E$

Some comments on Proposition 2 are in order. Although the polar case $\bar{e}_{1}=e_{1}^{0}$ does not qualify as climate policy, it is included in Proposition 2(i) because it will help understanding the rationale of cost-effective policies below. Proposition 2(i) confirms that there exists an intermediate non-empty interval $E_{m}$ of inputs $e_{A 1}$, if and only if the ceiling is strictly binding. $E_{m}$ is of special interest, because the ceiling policy is characterized by $\pi_{1}>0$ and $\pi_{2}<0$, if and only if the corresponding equilibrium exhibits $e_{A 1} \in E_{m}$ (see Proposition 2(ii)(c). An important insight is that $E_{m} \subset E$, if $\bar{e}_{1}<e_{1}^{0}$ and $e_{A 1}\left(\pi_{t}=0\right) \in E$ for $t=1,2$.

According to Proposition 2(ii) the tax rates $\pi_{1}$ and $\pi_{2}$ are both strictly decreasing in $e_{A 1}$ and that proposition also allows to determine the switches of these final instruments from taxes proper to subsidies. The information of Proposition 2(ii) is illustrated in Figure 1 and 2 for the case $\bar{e}_{1}<e_{1}^{0}$. The tax/subsidy switching points define the partition $E_{\ell}$, $\left\{e_{A 1}\left(\pi_{2}=0\right)\right\}, E_{m},\left\{\bar{e}_{1} / 2\right\}$ and $E_{h}$ of the interval $\left[0, \bar{e}_{1}\right]$ which we consider to be equal to the set $E$ of feasible policies for convenience of exposition. At low levels of $e_{A 1}$, i.e. for $e_{A 1} \in E_{\ell}$, the ceiling policy works via emission taxes proper, $\pi_{1}>0, \pi_{2}>0$; at intermediate 


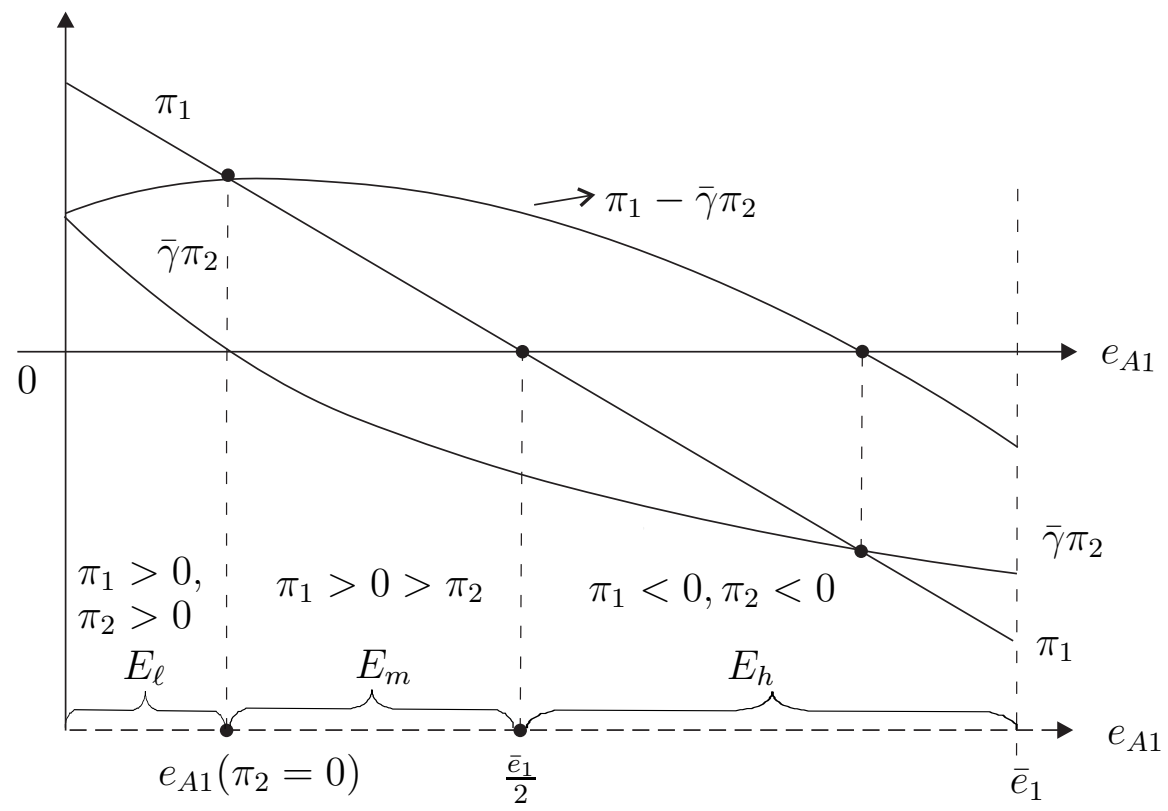

Figure 2: Classification of feasible unilateral ceiling policies

levels of $e_{A 1}$, i.e. for $e_{A 1} \in E_{m}$, we need a first-period tax, $\pi_{1}>0$, but a second-period subsidy, $\pi_{2}<0$; at high levels of $e_{A 1}$, i.e. for $e_{A 1} \in E_{h}$, the ceiling policy works via emission subsidies, $\pi_{1}<0, \pi_{2}<0$. To illustrate the limiting case $\bar{e}_{1}=e_{1}^{0}$, Figure 1 and 2 need to be modified such that the points $e_{A 1}\left(\pi_{1}=0\right)$ and $e_{A 1}\left(\pi_{2}=0\right)=\bar{e}_{1} / 2$ in Figure 1 and on the abscissa of Figure 2 collapse into one point, namely $e_{A 1}\left(\pi_{1}=0\right)=e_{A 1}\left(\pi_{2}=0\right)=\bar{e}_{1} / 2$. As a consequence, there are no feasible ceiling policies of the type $\left(\pi_{1}>0, \pi_{2}<0\right)$ anymore.

As established in Proposition 2(iii), $p_{e}$ is strictly increasing in $e_{A 1} \cdot p_{x}$ has also been shown to be strictly increasing in $e_{A 1}$ on $\left\{\left\{e_{A 1}\left(\pi_{2}=0\right)\right\}, E_{m},\left\{\bar{e}_{1} / 2\right\}\right\}$, but non-monotonicity for small and for large values of $e_{A 1}$ cannot be ruled out. If $p_{x}$ were increasing in $e_{A 1}$ on the total set $E$, the implication would be that all ceiling policies would be characterized by $\pi_{1}>\bar{\gamma} \pi_{2}$ - independent of the sign of their tax rates. Unfortunately, analytical complexity prevents proving that conjecture.

Another remarkable feature of feasible policies is that $e_{A 2}$ is increasing in $e_{A 1}$ (see Proposition 2(iii)). To see the implication, take as a point of departure a ceiling policy for the lowest possible level of $e_{A 1}$ in which country $A$ exports fossil fuel. As country $A$ 's resource stock $\alpha_{A} \bar{e}$ is given its exports of fossil fuel shrink with successive parametric increases in $e_{A 1}$ such that exports eventually turn into imports. Alternatively, if country $A$ has imported fossil fuel initially (e.g. in the case $\alpha_{A}=0$ ) its fossil fuel imports would expand. All these shifts are accompanied by rising prices of fossil fuel such that country $A$ 's export revenues shrink or its import bill rises. 
Proposition 2(iv) compares the prices $p_{e}$ and $p_{x}$ in ceiling policies with their counterparts in the laissez-faire economy. The fossil fuel price is declining in $e_{A 1}$ on the interval $\left.0, e_{A 1}\left(\pi_{2}=0\right)\right] \cap E$ under policies reducing the world demand for fossil fuel. As discussed above, it is unclear whether the price for second-period consumption, $p_{x}$ is monotone on that interval. If it also shrinks that would be equivalent to a price hike for first-period consumption because the latter is taken as numéraire. Note, however, that the interval $\left[0, e_{A 1}\left(\pi_{2}=0\right)\right]$ is rather small because $e_{A 1}\left(\pi_{2}=0\right)<\bar{e}_{1} / 2$. Ceiling policies on that interval do not involve subsidies.

What is the information Proposition 2 provides on the set $E$ of values of $e_{A 1}$ for which a ceiling policy exists? We know that $E \subset\left[0, \bar{e}_{1}\right]$ and that $E_{m} \subset E$ (Proposition 2(i)), but otherwise Proposition 2 characterizes ceiling policies for values of $e_{A 1} \in \bar{E}_{m}:=\left\{e_{A 1} \in\right.$ $\left.\left[0, \bar{e}_{1}\right] \mid e_{A 1} \notin E_{m}\right\}$ presupposing that $E \cap \bar{E}_{m} \neq \emptyset$. We prove now by means of a numerical example that $E \cap \bar{E}_{m}$ is non-empty, indeed. For the parameter values $a=0.2, b=0.1$, $\bar{e}=2, \bar{e}_{1}=0.95$ and $\gamma=0.5$ we calculate $e_{1}^{0}=1>\bar{e}_{1}=0.95, e_{A 1}\left(\pi_{2}=0\right)=0.270$, $e_{A 1}\left(\pi_{1}=0\right)=0.475=\bar{e}_{1} / 2$ and find that

$$
\left.\left.E=[0,0.921]=E_{\ell} \cup\{0.271\} \cup E_{m} \cup\{0.475\} \cup\right] 0.475,0.921\right] .
$$

It turns out that emissions $\left.\left.e_{A 1} \in\right] 0.921,0.95\right] \subset E_{h}$ are incompatible with a feasible ceiling policy because associated to $e_{A 1}=0.921$ is $e_{A 2}=1.079$ such that $e_{A 1}+e_{A 2}=2=\bar{e}$. Since $e_{A 2}$ is increasing in $e_{A 1}$ the market equilibrium condition of fossil fuel (3) is violated for values of $e_{A 1}$ larger than 0.921 . Nonetheless, the important message of the example is that $E \cap E_{\ell} \neq \emptyset$ and $E \cap E_{h} \neq \emptyset$. It is also worth noting that in this numerical example $p_{x}$ is strictly increasing in $e_{A 1}$ which implies $\pi_{1}>\bar{\gamma} \pi_{2}$ for all $e_{A 1} \in E$ and thus supports the conjecture that this property holds for all feasible policies. The associated price $p_{x}$ varies from 0.808 to 0.972 .

How do the allocative distortions generated by the unilateral policy compare to those of the cooperative policy that we have characterized in Proposition 1(ii)? Interestingly, consumption efficiency carries over to the unilateral policy, but in addition to the wedge between the marginal rates of intertemporal substitution in production and consumption at the unilateral policy the ratios of marginal productivities differ intra- and intertemporally and hence production efficiency is violated.

The specification of feasible unilateral ceiling policies presented here certainly is an interesting piece of information in its own right. However, since country A's welfare - and its cost of climate policy - varies with the policy chosen from the set of feasible ceiling policies, it is also of great interest to know which of those policies is country $A$ 's welfare 
maximizing - or cost-effective - policy. We turn to this issue in the following section.

\subsection{Cost-effective unilateral ceiling policies}

Suppose the government of country $A$ knows what we have established above that for each $e_{A 1} \in E$ there is a policy $\left(\pi_{1}, \pi_{2}\right)$ implementing the predetermined ceiling $\bar{e}_{1} \leq e_{1}^{0}$ given that the government of country $B$ refrains from climate policy. If it carries out the ceiling policy related to some $e_{A 1} \in E$, the representative consumer of country $A$ attains the utility

$$
u_{A}\left(e_{A 1}\right)=\left[x_{A 1}\left(e_{A 1}\right)\right]^{\gamma} \cdot\left[x_{A 2}\left(e_{A 1}\right)\right]^{1-\gamma},
$$

where we use here, temporarily only, the notation $x_{A 1}\left(e_{A 1}\right)$ and $x_{A 2}\left(e_{A 1}\right)$ to indicate that the levels of consumption $x_{A 1}$ and $x_{A 2}$ in (16) are those prevailing in the competitive equilibrium corresponding to the ceiling policy related to $e_{A 1} \in E$. Our subsequent analysis is based on the assumption that the utility (16) is single-peaked in $e_{A 1} \cdot{ }^{16}$

Invoking (15) we rewrite $(16)$ as $u_{A}\left(e_{A 1}\right)=\gamma^{\gamma}(1-\gamma)^{1-\gamma} p_{x}\left(e_{A 1}\right)^{\gamma-1} y_{A}\left(e_{A 1}\right)$. We differentiate $u_{A}$ with respect to $e_{A 1}$ and obtain

$$
\frac{\mathrm{d} u_{A}}{\mathrm{~d} e_{A 1}}=\frac{u_{A}}{y_{A}} \cdot \frac{\mathrm{d} y_{A}}{\mathrm{~d} e_{A 1}}-(1-\gamma) \frac{u_{A}}{p_{x}} \cdot \frac{\mathrm{d} p_{x}}{\mathrm{~d} e_{A 1}},
$$

where $^{17}$

$$
\begin{aligned}
\frac{\mathrm{d} y_{A}}{\mathrm{~d} e_{A 1}} & =\frac{b\left(\pi_{1}+\gamma \Delta e_{A}\right)}{\gamma}-x_{B 2}^{s} \frac{\mathrm{d} p_{x}}{\mathrm{~d} e_{A 1}} \\
\frac{\mathrm{d} p_{x}}{\mathrm{~d} e_{A 1}} & =\frac{b p_{x}\left(\pi_{1}-\bar{\gamma} \pi_{2}\right)}{\bar{\gamma}\left(p_{x} x_{2}^{s}-p_{e} \pi_{2}\right)}=\frac{b p_{x}\left(\pi_{1}-\bar{\gamma} \pi_{2}\right)}{x_{1}^{s}-\bar{\gamma} p_{e} \pi_{2}}
\end{aligned}
$$

with $\Delta e_{A}:=\alpha_{A} \bar{e}-e_{A 1}-e_{A 2}, \bar{\gamma}:=\frac{\gamma}{1-\gamma}$ and $x_{t}^{s}:=x_{A t}^{s}+x_{B t}^{s}$ for $t=1,2$. According to (17) the response of welfare to a small change in the ceiling policy (induced by $\mathrm{d} e_{A 1}$ ) is determined by the income effect (18) and the price effect (19). Since the tax rates $\pi_{1}$ and $\pi_{2}$ are not sign-constrained, the signs of these effects are unclear. We consider (15), (18) and (19) in (17) to get, after some rearrangement of terms,

$$
\frac{\mathrm{d} u_{A}}{\mathrm{~d} e_{A 1}}=\frac{u_{A}}{\gamma y_{A}}\left[\left(\pi_{1}+\gamma \Delta e_{A}\right)-\left(\pi_{1}-\bar{\gamma} \pi_{2}\right) \cdot G\right], \quad \text { where } \quad G:=\frac{\gamma p_{x}\left(x_{A 2}+x_{B 2}^{s}\right)}{x_{1}^{s}-\bar{\gamma} p_{e} \pi_{2}}>0 .
$$

Equation (20) gives rise to the following observations proved in the Appendix D.

\footnotetext{
${ }^{16}$ Our strong conjecture is that single-peakedness holds unconditionally but we have not been able to establish that analytically because several terms with opposite signs are involved. For the numerical example of Section 3.1 the single-peakedness assumption is satisfied.

${ }^{17}$ Differentiation of $y_{A}=\frac{x_{A 1}^{s}+x_{B 1}^{s}}{\gamma}-y_{B}$ yields $\mathrm{d} y_{A}=\frac{b \pi_{1}}{\gamma} \mathrm{d} e_{A 1}-\mathrm{d} y_{B}$. Inserting $(\mathrm{C} 7)$ of the Appendix $\mathrm{C}$ establishes (18). For the derivation of (19) see the proof of Proposition 2(iii) in the Appendix C.
} 


\section{Proposition 3.}

(i) Suppose $\bar{e}_{1}=e_{1}^{0}$. Country A's cost-effective ceiling policy belongs to the set

$$
\left\{\begin{array}{c}
E_{\ell} \\
\left\{\bar{e}_{1} / 2\right\} \\
E_{h}
\end{array}\right\} \text { with tax rates }\left\{\begin{array}{c}
\left(\pi_{1}>0, \pi_{2}>0\right) \\
\left(\pi_{1}=\pi_{2}=0\right) \\
\left(\pi_{1}<0, \pi_{2}<0\right)
\end{array}\right\} \text { if and only if } \alpha_{A}\left\{\begin{array}{l}
< \\
>
\end{array}\right\} 1 / 2 .
$$

(ii) Suppose $\bar{e}_{1}<e_{1}^{0}$.

(a) If $\alpha_{A} \leq 1 / 2$, country $A$ 's cost-effective ceiling policy belongs to the set $\left\{E_{\ell} \cup\right.$ $\left.\left\{e_{A 1}\left(\pi_{2}=0\right)\right\} \cup E_{m}\right\} \cap E$ and exhibits $\pi_{1}>0$. The sign of $\pi_{2}$ is unclear.

(b) If $\alpha_{A} \geq 1 / 2$ and $\gamma \geq 1 / 2$, country $A$ 's cost-effective ceiling policy belongs to the set $\left\{E_{m} \cup\left\{\bar{e}_{1} / 2\right\} \cup E_{h}\right\} \cap E$ and exhibits $\pi_{2}<0$. The sign of $\pi_{1}$ is unclear.

Proposition 3(i) takes up the limiting case $\bar{e}_{1}=e_{1}^{0}$ again and it demonstrates the link of the issue at hand with the standard theory of strategic environmental policy and international trade (Rauscher 1994, Ulph 1996). That link exists because carbon emissions are proportional to the consumption/burning of fossil fuel and because fossil fuel is traded on a world market. As a consequence, if country $A$ imports fuel and taxes (at a positive rate) its domestic fuel consumption, that tax is also levied on the amount of fuel imported, and to that extent the tax incidence is the same as that of an import tariff on fossil fuel. The tax diminishes the world demand for fossil fuel, ceteris paribus, and thus reduces the fossil fuel price because the global supply of fossil fuel is fixed. Country $A$ generates this termsof-trade effect intentionally to reduce its fossil-fuel import bill. Conversely, if country $A$ exports fossil fuel and taxes (at a negative rate) its domestic fuel consumption that negative tax is equivalent to an export subsidy on fossil fuel. It raises the world demand for fossil fuel, ceteris paribus, and thus raises the fossil fuel price given the fixed global supply of fossil fuel. That terms-of-trade effect increases country $A$ 's revenues from fossil fuel exports. In sum, the government of country $A$ chooses its policy in an effort to manipulate the terms-of-trade effect to enhance domestic welfare. For $\bar{e}_{1}=e_{1}^{0}$, the laissez-faire equilibrium with $e_{A 1}=e_{A 1}^{0}$ and $\pi_{1}=\pi_{2}=0$ clearly qualifies as a ceiling policy. But equation (20) then reads

$$
\left.\frac{\mathrm{d} u_{A}}{\mathrm{~d} e_{A 1}}\right|_{\bar{e}_{1}=e_{1}^{0}, \pi_{1}=\pi_{2}=0}=\frac{b u_{A}}{y_{A}} \Delta e_{A},
$$

which readily reveals the incentives of country $A$ 's government to deviate from the laissezfaire equilibrium. The government knows that it can do better by acting strategically and chooses a ceiling policy with emission subsidies in both periods which is associated with 
a higher [lower] level of $e_{A 1}$, if $\Delta e_{A}>0\left[\Delta e_{A}<0\right]$. If $\Delta e_{A}>0$, increasing $e_{A 1}$ raises $p_{e}$ and with it the fuel export revenues, ceteris paribus. However, this favorable price effect is eventually neutralized by a countervailing quantity effect. That quantity effect arises because we have established in Proposition 2 above that $e_{A 2}$ increases along with $e_{A 1}$ such that increasing $e_{A 1}$ diminishes the amount of fuel exported $\left(\Delta e_{A}\right)$. The government of country $A$ seeks to balance both effects to maximize domestic welfare. Analogous arguments apply to the case $\alpha_{A}<1 / 2$.

Proposition 3(ii) addresses the relevant case $\bar{e}_{1}<e_{1}^{0}$ and confirms that if country $A$ 's share of the fossil fuel stock is small cost effectiveness requires an emission tax proper in the first period $\left(\pi_{1}>0\right)$ while the cost effective second-period emission tax is negative $\left(\pi_{2}<0\right)$ if country $A$ owns a large share of the fossil fuel stock. We have argued in Section 3 that the simplest (and hence the relevant) fully cooperative policy to attain the unique cost-effective solution is to levy a uniform emission tax in period 1 and leave second-period emissions unregulated. In sharp contrast, country $A$ 's unilateral cost-effective ceiling policy generally consists in regulating domestic emissions in both periods and the tax rates shift from positive to negative with the size of country $A$ 's share of the fossil fuel stock. Which of the feasible policies is the cost-effective one only depends on the countries' fossil fuel endowments.

Although Proposition 3(ii) does not fully characterize country A's cost-effective policy, the principal message appears to be similar to that of the case of the weakly binding ceiling $\bar{e}_{1}=e_{1}^{0}$ in Proposition 3(i). When country $A$ 's fossil fuel stock is small [large] relative to that of country $B$, the cost-effective policy tends to be related to a relatively high [low] level of $e_{A 1}$. In other words, when country $A$ 's share of the world stock of fossil energy increases, country $A$ eventually turns into a fuel exporter whose strategic interest it is to either lower the positive emission tax or even subsidize rather than tax emissions. In both cases the domestic use of fossil energy is stimulated which in turn boosts the fossil fuel price and thus increases country $A$ 's fuel export revenues. To further substantiate that insight we rewrite the first-order condition for maximizing utility (20) in the following way:

$$
\frac{\mathrm{d} u_{A}}{\mathrm{~d} e_{A 1}}=0 \quad \Longleftrightarrow \quad F\left(e_{A 1} ; \alpha_{A}\right)=H\left(e_{A 1}\right)
$$

where

$$
\begin{aligned}
F\left(e_{A 1} ; \alpha_{A}\right) & :=\pi_{1}\left(e_{A 1}\right)+\gamma\left[\alpha_{A} \bar{e}-e_{A 1}-e_{A 2}\left(e_{A 1}\right)\right], \\
H\left(e_{A 1}\right) & :=\left[\pi_{1}\left(e_{A 1}\right)-\bar{\gamma} \pi_{2}\left(e_{A 1}\right)\right] \cdot G\left(e_{A 1}\right) .
\end{aligned}
$$

The term $G\left(e_{A 1}\right)$ defined in (20) depends on $e_{A 1}$ in a complicated way. For our purposes 
it suffices, however, to take advantage of the observation that under mild conditions ${ }^{18}$ $G\left(e_{A 1}\right) \in[0,1]$. In that case the graph of the function $H$ is plotted in Figure 3 as the winding curve in the area between the curve $\pi_{1}-\bar{\gamma} \pi_{2}$ and the $e_{A 1}$-axis. The function $F$ is strictly decreasing in ${ }^{19} e_{A 1}$ and has the property that its graph shifts upward with increasing $\alpha_{A}$. Figure 3 depicts four alternative graphs of $F$ for different shares $\alpha_{A}$. The greater the share $\alpha_{A}$ is, the further to the right is the graph of $F$. The cost-effective ceiling policy is determined by the intersection point of the graphs of $F$ and $H$. The straightforward implication is that the greater is country $A$ 's share $\alpha_{A}$ of the world stock of fossil fuel, the higher is the level of $e_{A 1}$ that characterizes the cost-effective ceiling policy. If for low shares $\alpha_{A}$ the cost-effective policy is in $E_{\ell}$, it moves into $E_{m}$ and likely further into $E_{h}$ with successively increasing $\alpha_{A}$. The information added by Figure 3 to the results of Proposition 3 is that the level of $e_{A 1}$ in the cost-effective policy rises smoothly with share $\alpha_{A}$. Unfortunately, however, Figure 3 does not provide rigorous conditions under which the cost-effective policy belongs to a specific subset of the interval $\left[0, \bar{e}_{1}\right]$.

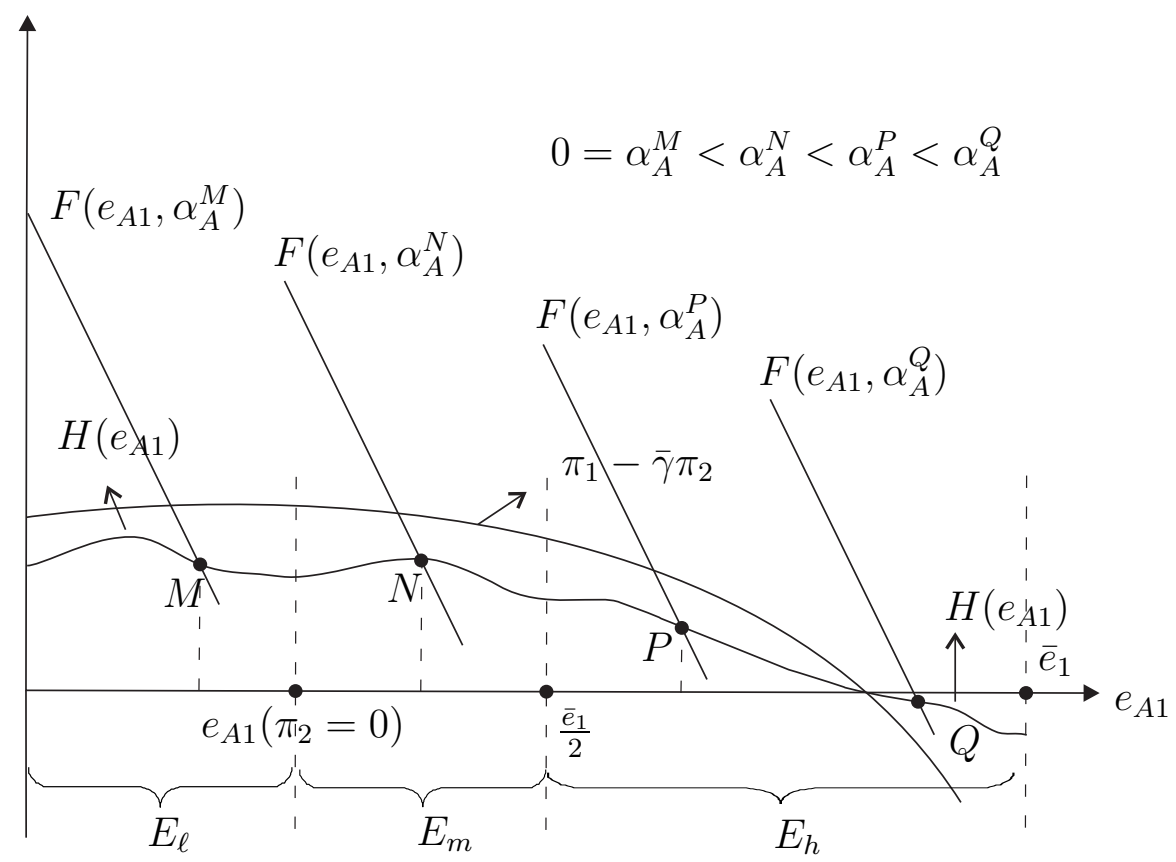

Figure 3: Cost-effective unilateral ceiling policies depending on country $A$ 's fossil-energy endowment

\footnotetext{
${ }^{18}$ For details on this constraint see the proof of Proposition 3(ii) in the Appendix D.

${ }^{19}$ Single-peakedness of the function $u_{A}$ from (16) requires that $F_{e_{A 1}}<H_{e_{A 1}}$ for all $e_{A 1}$. Although sign and magnitude of $H_{e_{A 1}}$ are unclear, the derivative $F_{e_{A 1}}=-2-\gamma\left(1+\frac{\mathrm{d} e_{A 2}}{\mathrm{~d} e_{A 1}}\right)<-(2+\gamma)$ is very small which is why $F_{e_{A 1}}<H_{e_{A 1}}$ for all $e_{A 1}$ is very likely.
} 


\section{Concluding remarks}

This paper builds on the proposition that reducing climate change damage requires curbing worldwide carbon emissions in the near to medium future (ceiling policy) and is therefore conceptually in line with the political goal of keeping the world mean temperature from rising $2^{\circ}$ Celsius or more above preindustrial levels. After more than two decades of international climate negotiations the prospects for a fully cooperative ceiling policy appear to be bleak. Nonetheless, several countries are taking action to abate domestic emissions and/or have announced to do so in the far future. But owing to free riding and carbon leakage, the net effects of such uncoordinated unilateral policies on global medium term emissions are unclear for the abating countries. That would be different if the 'willing countries' cooperate in a sub-global climate coalition, as we assume in the present paper. Although carbon leakage is still an issue in that case, such a coalition can implement some agreed upon medium-term global emission ceiling in joint action and can, conceptually at least, calculate the cost accruing to the coalition. As we have shown, even more important is that the coalition can choose from a set of policies that meet a given ceiling but differ in costs to be borne by the coalition. It will therefore seek to identify and choose that particular unilateral policy which minimizes its costs of implementing the agreed-upon ceiling.

We have characterized the unilateral feasible ceiling policies and the cost-effective policy and have compared that regulation with the fully cooperative cost-effective ceiling policy. We found that the unilateral cost-effective policy requires regulating the coalition's emissions in all periods while in case of full cooperation cost effectiveness can be attained through a ceiling policy consisting of a world-wide emission tax levied in the first period only that is uniform across countries. We also found that the greater is the coalition's share of the world stock of fossil energy the more likely it is that the cost-effective second period emission tax or even the taxes in both periods are negative, i.e. the unilateral costeffective policy may call for emission subsidies rather than emission taxes. When the climate coalition's share of the world stock of fossil energy increases it turns into an exporter of fossil fuel eventually and has an incentive to stimulate domestic fuel demand via emission subsidies in order to increase its fuel export revenues.

The price to be paid for the substantive analytical results achieved in the paper is simplifying assumptions. Our model consists of two periods and two countries only and applies, in addition, parametric functions for production and utility. The countries are alike except for their stock of fossil energy resources, and the negative climate externality being the raison d'être for climate policy is not contained in the formal model. Eichner and Pethig 
(2011) show that omitting consumption externalities, abatement technologies and multiple periods is not an essential restriction - although extending the model along these lines would prevent us from reaching meaningful results. Since it is the expected cost of climate policy that makes countries reluctant to take action the quest for cost effectiveness is indispensible for making progress in practical climate policy. Therefore, more work is desirable on the characterization of cost-effective sub-global ceiling policies. Although simple models like the model in the present paper are a necessary step on the way to a fuller understanding of unilateral climate policy, the restrictions one needs to impose for obtaining analytical results suggest that tractable analytical models should be supplemented by large-scale, less stylized models which are calibrated with realistic empirical data.

It should also be noted, however, that the strategic use of emission taxes identified and characterized in our simple analytical model will not be absent in cost-effective policies of sub-global climate coalitions in more complex settings including those where countries outside the coalition play Nash or those where stock-dependent extraction costs, low-carbon backstops etc. suggest climate policies that leave part of the fossil-fuel resources in the ground.

\section{References}

Ackerman, F. (2004), "Priceless benefits, costly mistakes: What's wrong with cost-benefit analysis?", Post-Autistic Economics Review 25

Allen, M. R., Frame, D. J., Huntingford, C., Jones, C. D., Lowe, J. A., Meinshausen, M., and Meinshausen, N. (2009), "Warming caused by cumulative carbon emissions towards the trillionth tonne", Nature 458, 1163-1166

Bohm, P. (1993), "Incomplete international cooperation to reduce $\mathrm{CO}_{2}$ emissions: Alternative policies", Journal of Environmental Economics and Management 24, 258-271

Chakravorty, U., Magne, B., and Moreaux, M. (2006), "A Hotelling model with a ceiling on the stock of pollution", Journal of Economic Dynamics and Control 30, 2875-2904

Copeland, B., and Taylor, M.S. (2005), "Free trade and global warming: A trade theory view of the Kyoto Protocol", Journal of Environmental Economics and Management $49,205-234$

Council of the European Union (2005), European Council, Brussels, 22 and 23 March 2005, Presidential conclusions 7619/05 REV1 
Di Maria, C., and van der Werf, E. (2008), "Carbon leakage revisited: Unilateral climate policy with directed technical change", Environmental and Resource Economics 39, $55-74$

Eichner, T., and Pethig, R. (2011), "Carbon leakage, the green paradox and perfect future markets", International Economic Review, forthcoming

Gerlagh, R. (2011), "Too much oil", CESifo Economic Studies 57, 25-43

Golombek, R., and Hoel, M. (2004), "Unilateral emission reductions and cross-country spillovers", The B.E. Journal of Economic Analysis and Policy 0(2)

Grafton, R. Q., van Long, N., and Kompas, T. (2010), "Biofuels subsidies and the green paradox", CESifo Working Paper No. 2960

Hoel, M. (1991), "Global environmental problems: The effects of unilateral actions taken by one country", Journal of Environmental Economics and Management 20, 55-70

Hoel, M. (2010), "Climate change and carbon tax expectations", CESifo Working Paper No. 2966.

Ishikawa, J., and Kiyono, K. (2006), "Greenhouse-gas emissions control in an open economy", International Economic Review 47, 431-450

Kalkuhl, M., and Edenhofer, O. (2010), "Prices vs. quantities and the intertemporal dynamics of the climate rent", Conference Paper WCERE 2010

Lave, L. (1996), "Benefit cost analysis: Do the benefits exceed the costs?" in: R.W. Hahn, Cost and Lifes Saved: Getting Better Results from Regulation, Oxford University Press, 104-134

Meinshausen, M., Meinshausen, N., Hare, W., Raper, S.B.C., Frieler, K., Knutti, R., Frame, D.J., and Allen, M.R. (2009), "Greenhouse-gas emission targets for limiting global warming to $2^{\circ} \mathrm{C}^{\prime \prime}$, Nature $458,1158-1163$

Michielsen, T. (2011), "Brown backstops versus the green paradox", CentER discussion paper No. 2011-076.

Rauscher, M. (1994), "On ecological dumping", Oxford Economic Papers 46, 822-840

Sinclair, P. (1992), "High does nothing and rising is worse: Carbon taxes should be kept declining to cut harmful emissions", Manchester School 60, 41-52

Sinclair, P. (1994), "On the optimum trend of fossil fuel taxation", Oxford Economic Papers 46, 869-877 
Sinn, H.-W. (2008), "Public policies against global warming: A supply side approach", International Tax and Public Finance 15, 360-394

Ulph, A. (1997), "International trade and the environment: A survey of recent economic analysis", in: H. Folmer and T. Tietenberg (eds.), The International Yearbook of Environmental and Resource Economics 1997/1998, Edward Elgar, Cheltenham UK UNFCCC (2009), 'Decision-/CP15', Copenhagen Accord, Denmark (December 18)

UNFCCC (2010), ), United Nations Framework Convention on Climate Change, Cancun

Van der Ploeg, F., and Withagen, C. (2010), "Is there really a green paradox?", CESifo Working Paper No. 2963

WBGU (2009), Kassensturz für den Weltklimavertrag - Der Budgetansatz, Sondergutachten

Weitzmann, M. L. (2009), "On modeling and interpreting the economics of catastrophic climate change", Review of Economics and Statistics 91, 1-19

\section{Appendix}

List of notation introduced to simplify the exposition:

$$
\begin{aligned}
& \left.\Delta e_{i}:=\alpha_{i} \bar{e}-e_{i 1}-e_{i 2} i=A, B, \quad G:=\frac{\gamma p_{x}\left(x_{A 2}+x_{B 2}^{s}\right)}{x_{1}^{s}-\bar{\gamma} p_{e} \pi_{2}}, \quad \bar{\gamma}:=\frac{\gamma}{1-\gamma}, \gamma \in\right] 0,1[, \\
& \pi_{1}:=\frac{\pi_{A 1}}{b}, \pi_{2}:=\frac{\pi_{A 2}}{b p_{x}}, \quad x_{t}^{s}:=x_{A t}^{s}+x_{B t}^{s}, \quad x_{t}^{s *}:=a \bar{e}_{t}-\frac{b \bar{e}_{t}^{2}}{4}, t=1,2 .
\end{aligned}
$$

$e_{A 1}\left(\pi_{t}=0\right)$ is the value of $e_{A 1}$ that leads to ceiling policy which exhibits $\pi_{t}=0$ for $t=1,2$. $p_{x}\left(e_{A 1}\right)$ is the price $p_{x}$ that prevails in the ceiling-policy equilibrium for $e_{A 1} \in E$. We use analogous notation for equilibrium values of other variables, e.g. $x_{A 2}\left(e_{A 1}\right)$ etc. Letters with superscript "0", e.g. $e_{1}^{0}$, denote the value of the respective variable in the 'benchmark' competitive laissez-faire equilibrium.

\section{A. On the market for savings and the choice of numéraires}

Consider the following subset of equations of the model of Section 2:

$$
\begin{array}{rlrl}
e_{2} & =\bar{e}-\bar{e}_{1}=e_{A 2}+e_{B 2}, & \\
x_{A t}^{s}+x_{B t}^{s} & =x_{A t}+x_{B t} & & t=1,2, \\
y_{i} & :=x_{i 1}^{s}+p_{x} x_{i 2}^{s}+p_{e}\left(\alpha_{i} \bar{e}-e_{i 1}-e_{i 2}\right) & & i=A, B, \\
y_{i} & =x_{i 1}+p_{x} x_{i 2} & i=A, B .
\end{array}
$$


The equations (A3) and (A4) presuppose $r=0$. The equations (A4) represent the countries' consolidated ('present value') budget constraints in which the information about incomes and expenditures per period is lost. That information is provided by defining the period incomes (=recycled profits)

$$
\begin{array}{ll}
y_{i 1}=x_{i 1}^{s}-p_{e} e_{i 1}+\alpha_{i} p_{e} e_{1} & i=A, B, \\
y_{i 2}=p_{x} x_{i 2}^{s}-p_{e} e_{i 2}+\alpha_{i} p_{e} e_{2} & i=A, B
\end{array}
$$

and the period budget constraints

$$
\begin{aligned}
y_{i 1} & =s_{i}+x_{i 1} & & i=A, B, \\
y_{i 2}+(1+r) s_{i} & =p_{x} x_{i 2} & & i=A, B .
\end{aligned}
$$

$s_{i}$ are the sign-unconstrained savings of country $i$ and $r$ is the market interest rate. The credit market equilibrium requires

$$
s_{A}+s_{B}=0
$$

The 9 new equations (A5) - (A9) contain the 7 new variables $y_{A 1}, y_{B 1}, y_{A 2}, y_{B 2}, s_{A}, s_{B}, r$ that are not in the model of Section 2. If the modification (A5) - (A9) of the model of Section 2 is adopted, the 4 equations (A3) and (A4) as well as the 2 variables $y_{A}$ and $y_{B}$ drop out. Ultimately that leaves us with 5 new variables and 5 new equations. However, adding the equation (6) for $i=1$ to the equation (A6) for $i=2$ yields, after some rearrangement of terms,

$$
p_{x}\left(x_{A 2}^{s}+x_{B 2}^{s}-x_{A 2}-x_{B 2}\right)+p_{e}\left(e_{2}-e_{A 2}-e_{B 2}\right)+(1+r)\left(s_{A}+s_{B}\right)=0 .
$$

It is straightforward that (A1), (A2) and (A10) imply (A9). Hence (A9) is redundant and thus provides the degree of freedom - and the necessity - to fix one price in addition to the price for the consumption good in period 1 which is already normalized $\left(p_{x 1} \equiv 1\right)$. That degree of freedom is often used to set $p_{x}=p_{x 2} \equiv 1$. In the present paper we choose $r \equiv 0$, instead. It is easy to see that with this normalization the present value of income, $y_{i 1}+y_{i 2} /(1+r)$, coincides with (A3) and the consolidated budget constraint $y_{i 1}+y_{i 2} /(1+r)+s_{i}=s_{i}+x_{i 1}+p_{x} x_{i 2} /(1+r)$ coincides with (A4).

\section{B. Cooperative cost-effective carbon ceiling regulation}

The Lagrangean corresponding to the problem of maximizing $\sum_{i=A, B} \omega_{i} u_{i}$ subject to (1) (5) reads

$$
\begin{aligned}
\mathcal{L} & =\sum_{i=A, B} \omega_{i} U^{i}\left(x_{i 1}, x_{i 2}\right)+\sum_{t=1,2} \lambda_{x t}\left[X^{A}\left(e_{A t}\right)+X^{B}\left(e_{B t}\right)-x_{A t}-x_{B t}\right] \\
& +\lambda_{e}\left(\bar{e}-e_{A 1}-e_{A 2}-e_{B 1}-e_{B 2}\right)+\bar{\lambda}\left(\bar{e}_{1}-e_{A 1}-e_{B 1}\right) .
\end{aligned}
$$


The first-order conditions of solving (B1) yield

$$
\begin{aligned}
\frac{U_{x_{i 2}}}{U_{x_{i 1}}} & =\mu_{x 2} & & \text { for } \quad i=A, B, \\
X_{e_{i 1}} & =\mu_{e}+\bar{\mu} & & \text { for } \quad i=A, B, \\
\mu_{x 2} X_{e_{i 2}}^{i} & =\mu_{e} & & \text { for } \quad i=A, B,
\end{aligned}
$$

where $\mu_{x 2}:=\lambda_{x 2} / \lambda_{x 1}, \mu_{e}:=\lambda_{e} / \lambda_{x 1}$ and $\bar{\mu}:=\bar{\lambda} / \lambda_{x 1}$ are positive shadow prices in terms of first-period output $X$ evaluated at the solution of (B1). To make use of the information contained in (B2) - (B4) about the efficient allocation in the standard procedure of decentralization by prices (and taxes), denote a cooperative tax policy as $\pi:=\left(\pi_{A 1}, \pi_{A 2}, \pi_{B 1}, \pi_{B 2}\right)$ and define the set

$$
\left.\left.\Pi:=\left\{\pi \in \mathbb{R}^{4} \mid \pi_{A 1}=\pi_{B 1}, \pi_{A 2}=\pi_{B 2}, \pi_{A 1}=\bar{\mu}+\pi_{A 2}, \pi_{A 2} \in\right]-\infty, \mu_{e}\right]\right\} .
$$

It is easy to see that with the definitions $\pi \in \Pi, p_{e}=\mu_{e}-\pi_{A 2}$ and $p_{x}=\mu_{x 2}$ the conditions (B2), (B3) and (B4) coincide with the conditions (7), (8) and (9). This is true for all $\pi \in \Pi$ which implies, in particular, that the allocation of inputs and outputs is the same for all $\pi \in \Pi$. Hence world income is uniquely determined. In the social planner's solution the market clearing conditions (4) are satisfied. Therefore, there exist incomes, say $y_{A}^{*}$ and $y_{B}^{*}$, satisfying $y_{A}^{*}+y_{B}^{*}=x_{A 1}^{s}+x_{B 1}^{s}+p_{x}\left(x_{A 2}^{s}+x_{B 2}^{s}\right)$, such that the consumption bundles $\left(x_{i 1}, x_{i 2}\right)$ in the solution of (B1) maximize utility $U^{i}(\cdot)$ subject to the income $y_{i}^{*}$. However, since $p_{e}$ is not invariant with respect to the choice of $\pi \in \Pi, y_{i}$ from (6) depends on $\pi$. We account for that relationship by writing $y_{i}=y_{i}(\pi)$ and observe that $y_{i}(\pi) \neq y_{i}^{*}$, in general. To assign the incomes $y_{A}^{*}$ and $y_{B}^{*}$ to the countries $A$ and $B$, respectively, we define $T(\pi):=y_{A}(\pi)-y_{A}^{*}$ and let country $A$ transfer the (positive or non-positive) amount $T(\pi)$ of its income $y_{A}(\pi)$ to country $B$.

To sum up, the cooperative tax policy $\pi=\left(\pi_{A 1}, \pi_{A 2}, \pi_{B 1}, \pi_{B 2}\right)$ implements the ceiling $\bar{e}_{1}$ cost-effectively, if and only if $\pi \in \Pi$. In the associated ceiling-policy equilibrium the prices are ${ }^{20} p_{e}=\mu_{e}-\pi_{A 2}$ and $p_{x}=\mu_{x 2}$. Moreover, country $A$ needs to make a suitable income transfer to country $B$ whose sign and magnitude depends on $\pi_{A 2},\left(\omega_{A}, \omega_{B}\right)$ and on $\left(\alpha_{A}, \alpha_{B}\right)$. The equilibrium allocation is the same for all $\pi \in \Pi$ and is characterized by

$$
\begin{array}{ll}
\frac{X_{e_{A 1}}^{A}}{X_{e_{B 1}}^{B}}=\frac{X_{e_{A 2}}^{A}}{X_{e_{B 2}}^{B}}=1, \frac{X_{e_{A 1}}^{A}}{X_{e_{A 2}}^{A}}=\frac{X_{e_{B 1}}^{B}}{X_{e_{B 2}}^{B}}=\left(1+\frac{\bar{\mu}}{\mu_{e}}\right) p_{x}, & \text { (production efficiency) } \\
\frac{U_{x_{A 2}}^{A}}{U_{x_{A 1}}^{A}}=\frac{U_{x_{B 2}}^{B}}{U_{x_{B 1}}^{B}}=p_{x}, & \text { (consumption efficiency) } \\
\frac{U_{x_{i 2}}^{i}}{U_{x_{i 1}}^{i}}-\frac{X_{e_{i 1}}^{i}}{X_{e_{i 2}}}=-\frac{\bar{\mu} p_{x}}{\mu_{e}} & \text { for } i=A, B .
\end{array}
$$

\footnotetext{
${ }^{20} p_{e}=\mu_{e}-\pi_{A 2} \geq 0$ is secured by the condition $\left.\left.\pi_{A 2} \in\right]-\infty, \mu_{e}\right]$ in the definition of the set $\Pi$.
} 


\section{Proof of Proposition 2}

Since the proofs of different parts of Proposition 2 are interrelated, the subsequent proof does not follow the sequence of results as listed in Proposition 2.

Proposition 2(iii), first sentence: To determine how ceiling policies differ in their respective equilibria, we will leave the ceiling $\bar{e}_{1}$ unchanged, disturb the initial equilibrium by a small (exogenous) variation in $e_{A 1}$, and then determine the displacement effects characterizing the new competitive equilibrium reached after the shock. Total differentiation of the equations (4) for $t=1,(6),(13),(14)$ and (15) leads to

$$
\begin{array}{lr}
\mathrm{d} \pi_{i}=-2 \mathrm{~d} e_{A i} & i=A, B, \\
\mathrm{~d} p_{e}=b \mathrm{~d} e_{A 1}, & \\
\frac{p_{e}}{p_{x}} \mathrm{~d} p_{x}+b p_{x} \mathrm{~d} e_{A 2}=\mathrm{d} p_{e}, & i=A, B, \\
\mathrm{~d} x_{i 1}=\gamma \mathrm{d} y_{i} & i=A, B, \\
\mathrm{~d} x_{i 2}=\frac{x_{i 2}}{y_{i}} \mathrm{~d} y_{i}-\frac{x_{i 2}}{p_{x}} \mathrm{~d} p_{x} & \\
\mathrm{~d} y_{A}=b \pi_{1} \mathrm{~d} e_{A 1}+b p_{x} \pi_{2} \mathrm{~d} e_{A 2}+\Delta e_{A} \mathrm{~d} p_{e}+x_{A 2}^{s} \mathrm{~d} p_{x}, & \\
\mathrm{~d} y_{B}=-\Delta e_{A} \mathrm{~d} p_{e}+x_{B 2}^{s} \mathrm{~d} p_{x}, & \\
\pi_{1} \mathrm{~d} e_{A 1}=\mathrm{d} x_{A 1}+\mathrm{d} x_{B 1} . &
\end{array}
$$

$\frac{\mathrm{d} \pi_{1}}{\mathrm{~d} e_{A 1}}=-2<0$ and $\frac{\mathrm{d} p_{e}}{\mathrm{de} e_{A 1}}=b>0$ are obvious from $(\mathrm{C} 1)$ and $(\mathrm{C} 2)$. Next insert $\mathrm{d} p_{e}$ from $(\mathrm{C} 2)$ into (C3) and consider the equations (C4), (C5) and (C7) in (C8) to obtain

$$
\frac{p_{e}}{p_{x}} \mathrm{~d} p_{x}+b p_{x} \mathrm{~d} e_{A 2}=b \mathrm{~d} e_{A 1} \quad \text { and } \quad \bar{\gamma} x_{2}^{s} \mathrm{~d} p_{x}+b \bar{\gamma} p_{x} \pi_{2} \mathrm{~d} e_{A 2}=b \pi_{1} \mathrm{~d} e_{A 1} .
$$

Solving these two equations for $\mathrm{d} p_{x}$ and $\mathrm{d} e_{A 2}$ yields

$$
\frac{\mathrm{d} p_{x}}{\mathrm{~d} e_{A 1}}=\frac{b p_{x}\left(\pi_{1}-\bar{\gamma} \pi_{2}\right)}{\bar{\gamma}\left(p_{x} x_{2}^{s}-p_{e} \pi_{2}\right)}=\frac{b p_{x}\left(\pi_{1}-\bar{\gamma} \pi_{2}\right)}{x_{1}^{s}-\bar{\gamma} p_{e} \pi_{2}}
$$

and $\frac{\mathrm{d} e_{A 2}}{\mathrm{~d} e_{A 1}}=\frac{x_{1}^{s}-p_{e} \pi_{1}}{p_{x}\left(x_{1}^{s}-\bar{\gamma} p_{e} \pi_{2}\right)}=\frac{x_{1}^{s}-p_{e} \pi_{1}}{\bar{\gamma} p_{x}\left(p_{x} x_{2}^{s}-p_{e} \pi_{2}\right)}$. Note that $\pi_{2}=e_{B 2}-e_{A 2}$ follows from (13) and (14) such that $p_{x} x_{2}^{s}-p_{e} \pi_{2}=p_{x} x_{A 2}^{s}+p_{e} e_{A 2}+p_{x} x_{B 2}^{s}-p_{e} e_{B 2}>0$, because the profit $p_{x} x_{B 2}^{s}-p_{e} e_{B 2}$ is positive. Likewise, $x_{1}^{s}-p_{e} \pi_{1}>0$ and therefore $\mathrm{d} e_{A 2} / \mathrm{d} e_{A 1}>0$. From (C1) and $\mathrm{d} e_{A 2} / \mathrm{d} e_{A 1}>0$ follows $\frac{\mathrm{d} \pi_{2}}{\mathrm{de} e_{A 1}}=-2 \frac{\mathrm{d} e_{A 2}}{\mathrm{~d} e_{A 1}}<0$.

Proposition 2(ii)(b). $\pi_{1}$ and $\pi_{2}$ have been shown to be strictly decreasing in $e_{A 1}$ over $E$ in the proof of the first sentence of Proposition 2(iii) above. We proceed in several steps.

Lemma 1. $\quad \bar{e}_{1}\left\{\begin{array}{l}< \\ =\end{array}\right\}$ implies $\frac{x_{1}^{s *}}{x_{2}^{s *}}\left\{\begin{array}{l}< \\ =\end{array}\right\} \frac{x_{1}^{s 0}}{x_{2}^{s 0}}=\bar{\gamma} p_{x}^{0}$ with $x_{t}^{s *}=a \bar{e}_{t}-b \frac{\bar{e}_{t}^{2}}{4}$ and $x_{t}^{s}=x_{A t}^{s}+x_{B t}^{s}$. 
Proof. Observe first that the equations (4), (6) and (15) imply

$$
x_{1}^{s}=\bar{\gamma} p_{x} x_{2}^{s}
$$

which holds in laissez-faire as well as with ceiling regulation. In the latter case we have

$$
\begin{aligned}
x_{t}^{s} & =a \bar{e}_{t}-\frac{b}{2} \bar{e}_{t}^{2}+b e_{A t} e_{B t}=a \bar{e}_{t}-\frac{b}{2} \bar{e}_{t}^{2}-\frac{b}{4} \bar{e}_{t}^{2}+\frac{b}{4} \bar{e}_{t}^{2}+b e_{A t} e_{B t} \\
& =x_{t}^{s *}-b\left(\frac{\bar{e}_{t}^{2}}{4}-e_{A t} e_{B t}\right) .
\end{aligned}
$$

$x_{t}^{s *}$ is the maximum possible production in $t$ under the constraint $\bar{e}_{t}$ which is attained if and only if $e_{A t}=e_{B t}$ or, equivalently, if and only if $\pi_{t}=0$. Lemma 1 is verified by observing that $x_{1}^{s *}<x_{1}^{s 0}$ and $x_{2}^{s *}>x_{2}^{s 0}$ if $\bar{e}_{1} \leq e_{1}^{0}$, and $x_{1}^{s *}=x_{1}^{s 0}$ and $x_{2}^{s *}=x_{2}^{s 0}$ if $\bar{e}_{1}=e_{1}^{0}$.

Lemma 2. If $\left(\pi_{1}, \pi_{2}=0\right)$ is a ceiling policy for $\bar{e}_{1}\left\{\begin{array}{l}< \\ =\end{array}\right\} e_{1}^{0}$, then $\bar{\gamma} p_{x}^{0}\left\{\begin{array}{l}> \\ =\end{array}\right\} \frac{x_{1}^{s}}{x_{2}^{s}}=\bar{\gamma} p_{x}$.

Proof. In the economy with ceiling regulation, the commodity market equilibria require (as shown above) $\frac{x_{1}^{s}}{x_{2}^{s}}=\frac{x_{1}^{s *}-b\left(\frac{\bar{e}_{1}^{2}}{4}-e_{A 1} e_{B 1}\right)}{x_{2}^{s *}-b\left(\frac{\bar{e}_{2}^{2}}{4}-e_{A 2} e_{B 2}\right)}$. As $\pi_{2}=0$ implies $e_{A 2}=e_{B 2}=\bar{e}_{2} / 2, x_{2}^{s}=x_{2}^{s *}$ follows. Moreover, $\left(\pi_{1}=0, \pi_{2}=0\right)$ is a ceiling policy, if and only if $\bar{e}_{1}=e_{1}^{0}$. Otherwise we must have $\pi_{1} \neq 0$ and hence $x_{1}^{s}<x_{1}^{s *}$. Combined with Lemma 1 these findings prove Lemma 2.

Lemma 3. If $\left(\pi_{1}, \pi_{2}=0\right)$ is a ceiling policy for $\bar{e}_{1}<e_{1}^{0}$ then $\pi_{1}>0$.

Proof. Contrary to the claim suppose that $\left(\pi_{1} \leq 0, \pi_{2}=0\right)$ is a ceiling policy. In that case we have $p_{e}=a-b e_{B 1}=p_{x}\left(a-b \bar{e}_{2} / 2\right), e_{B 1} \leq \bar{e}_{1} / 2$ and therefore $p_{x} \geq \frac{2 a-b \bar{e}_{1}}{2 a-b \bar{e}_{2}}$. In the laissez-faire economy we calculate $p_{x}^{0}=\frac{2 a-b e_{1}^{0}}{2 a-b e_{2}^{0}}$ which is smaller than $\frac{2 a-b \bar{e}_{1}}{2 a-b \bar{e}_{2}}$ because of $\bar{e}_{1}<e_{1}^{0}$. We conclude that $p_{x}^{0}=\frac{2 a-b e_{1}^{0}}{2 a-b e_{2}^{0}}<\frac{2 a-b \bar{e}_{1}}{2 a-b \bar{e}_{2}} \leq p_{x}$. However, $p_{x}<p_{x}^{0}$ follows from Lemma 2. That contradiction proves Lemma 3 and thus Proposition 2(ii)(b).

Proposition 2(i). Suppose first that $\bar{e}_{1}<e_{1}^{0}$ and observe that $e_{A 1}\left(\pi_{2}=0\right)<\bar{e}_{1} / 2$ holds because $\pi_{1}>0$ at $e_{A 1}\left(\pi_{2}=0\right)$ according to Proposition 2(ii)(b) and because $\pi_{1}$ is strictly decreasing in $e_{A 1}$ according to $(\mathrm{C} 1)$. Hence the level of $e_{A 1}$ at which $\pi_{1}$ becomes zero is greater than $e_{A 1}\left(\pi_{2}=0\right)$. In fact, we have $e_{A 1}\left(\pi_{1}=0\right)=\bar{e}_{1} / 2>e_{A 1}\left(\pi_{2}=0\right)$ which proves $E_{m} \neq \emptyset$. From $\bar{e}_{1}=e_{1}^{0}$ follows $e_{A 1}\left(\pi_{1}=0\right)=e_{A 1}\left(\pi_{2}=0\right)=\bar{e}_{1} / 2$ and therefore $E_{m}=\emptyset$.

Proposition 2(ii) cont'd. We have shown in the proof of the first sentence of Proposition 2(iii) above that $\pi_{1}$ and $\pi_{2}$ are strictly decreasing over the entire interval $E$. When 
combined with the result of Proposition 2(ii)(b), that observation completes the proof of Proposition 2(ii).

Proposition 2(iii), second sentence. According to Proposition 2(ii)(c), ceiling policies for $e_{A 1}$ over the interval $E_{m}$ are characterized by $\pi_{1}>0$ and $\pi_{2}<0$. That implies $\pi_{1}>\bar{\gamma} \pi_{2}$ and $\mathrm{d} p_{x} / \mathrm{d} e_{A 1}>0$ via $(\mathrm{C} 9)$.

Proposition 2(iv). Consider first the policy $\left(\pi_{1}>0, \pi_{2}=0\right)$ and observe that $e_{B 1}>$ $\bar{e}_{1} / 2>e_{A 1}$ owing to $\pi_{1}>0$. In view of (14) that leads to $p_{e}=a-b e_{B 1}<a-b\left(\bar{e}_{1} / 2\right)<$ $a-b\left(e_{1}^{0} / 2\right)=p_{e}^{0} \cdot p_{x}<p_{x}^{0}$ follows from Lemma 2 . We have established that $p_{x}$ and $p_{e}$ are increasing in $e_{A 1}$ over $E_{\ell}$. That completes the proof of Proposition 2(iv).

\section{Proof of Proposition 3}

Proposition 3(i). For $\pi_{1}=\pi_{2}=0$ and $\bar{e}_{1}=e_{1}^{0}$ we are in the laissez-faire equilibrium. The only way the countries $A$ and $B$ may differ from each other are differing fossil-fuel ownership shares $\alpha_{A}$ and $\alpha_{B}$. If $\alpha_{A}=\alpha_{B}$ no trade in fossil fuel and the commodity takes place $\left(\Delta e_{A}=0\right)$. Since the fuel demands are $e_{A t}=e_{B t}=\bar{e}_{t} / 2$ for $t=1,2$, it is straightforward that in the laissez-faire equilibrium we have $\Delta e_{A} \gtrless$ if and only if $\alpha_{A} \gtrless \alpha_{B}$. Combining this information with the single-peakedness of (16) and with $\left.\frac{\mathrm{d} u_{A}}{\mathrm{de} e_{A 1}}\right|_{\pi_{1}=\pi_{2}=0, \bar{e}_{1}=e_{1}^{0}}=\frac{b u_{A}}{y_{A}} \Delta e_{A}$ from (20) completes the proof.

Proposition 3(ii)(a). Consider the feasible ceiling policy $\left(\pi_{1}=0, \pi_{2}<0\right)$ as point of departure. In the corresponding equilibrium, country $A$ 's fossil fuel consumptions are $e_{A 1}=\bar{e}_{1} / 2$ and $e_{A 2}>\bar{e}_{2} / 2$ and hence $e_{A 1}+e_{A 2}>\bar{e} / 2$. From the presupposition $\alpha_{A} \leq 1 / 2$ follows $\Delta e_{A}<0$ such that (20) implies

$$
\frac{\mathrm{d} u_{A}}{\mathrm{~d} e_{A 1}}=\frac{u_{A}}{\gamma y_{A}}\left(\gamma \Delta e_{A}+\bar{\gamma} \pi_{2} G\right)<0 .
$$

Single-peakedness of $u_{A}$ in $e_{A 1}$ then establishes Proposition 3(ii)(a).

Proposition 3(ii)(b). Now we take the feasible ceiling policy $\left(\pi_{1}>0, \pi_{2}=0\right)$ as point of departure. In the corresponding equilibrium, country $A$ 's fossil-fuel consumptions are $e_{A 1}<\bar{e}_{1} / 2$ and $e_{A 2}=\bar{e}_{2} / 2$ and hence $e_{A 1}+e_{A 2}<\bar{e} / 2$. From the presupposition $\alpha_{A} \geq 1 / 2$ follows $\Delta e_{A}>0$ such that (20) implies

$$
\frac{\mathrm{d} u_{A}}{\mathrm{~d} e_{A 1}}=\frac{u_{A}}{\gamma y_{A}}\left[\pi_{1}(1-G)+\gamma \Delta e_{A}\right]>0 .
$$

Single-peakedness of $u_{A}$ in $e_{A 1}$ establishes Proposition 3(ii)(b) if $G \in[0,1]$. By definition, 


$$
\begin{aligned}
G:=\frac{\gamma p_{x}\left(x_{A 2}+x_{B 2}^{s}\right)}{x_{1}^{s}-\bar{\gamma} p_{e} \pi_{2}} \text { and } G & =\frac{(1-\gamma) p_{x}\left(x_{A 2}+x_{B 2}^{s}\right)}{p_{x} x_{2}^{s}} \text { since } \pi_{2}=0 \text {. Therefore } \\
G<1 & \Longleftrightarrow(1-\gamma) p_{x}\left(x_{A 2}+x_{B 2}^{s}\right)<p_{x} x_{2}^{s} \\
& \Longleftrightarrow 0<p_{x}\left[\gamma\left(x_{A 2}^{s}+x_{B 2}^{s}\right)+(1-\gamma)\left(x_{A 2}^{s}-x_{A 2}\right)\right] \\
& \Longleftrightarrow 0<p_{x}\left[\gamma\left(x_{A 2}+x_{B 2}\right)+(1-\gamma)\left(x_{A 2}^{s}-x_{A 2}\right)\right] \\
& \Longleftrightarrow 0<(2 \gamma-1) p_{x} x_{A 2}+p_{x}\left[\gamma x_{B 2}+(1-\gamma) x_{A 2}^{s}\right] \\
& \Longleftrightarrow \gamma>\frac{1}{2}-\frac{\gamma x_{B 2}+(1-\gamma) x_{A 2}^{s}}{2 x_{A 2}} .
\end{aligned}
$$

The right side of the last inequality is less than $1 / 2$ and may even be negative. Hence the qualification $\gamma \geq 1 / 2$ in Proposition 3(ii)(b) is a very weak sufficient condition. 